\title{
Educação continuada em unidade de terapia intensiva: revisão da literatura
}

\section{Continuous education in intensive care unit-icu: literature review}

\author{
Laurindo Pereira de Souza ${ }^{1}$, Marcia Guerino de Lima²
}

1. Mestrando em Ciências da Saúde pelo Instituto de Assistência Médica ao Servidor Público Estadual de São Paulo (IAMSPE) e Docente do Departamento de Enfermagem da Faculdade de Ciências Biomédicas (FACIMED), Cacoal, Rondônia, Brasil. 2. Docente do Departamento de Enfermagem da Faculdade de Ciências Biomédicas (FACIMED), Cacoal, Rondônia, Brasil.

\begin{abstract}
Resumo
Introdução: A educação continuada é um processo dinâmico de ensino-aprendizagem, ativo e permanente destinado a atualizar e melhorar a capacitação de pessoas. Objetivo: Este estudo objetivou fazer uma revisão bibliográfica acerca da importância da educação continuada em unidade de terapia intensivaUTI, revelar o ano dos artigos publicados, autores e os Estados em que foram publicados. Métodos: Trata-se de um estudo quantiqualitativo, descritivo realizado por meio de revisão bibliográfica publicada nos bancos de dados: LILACS (Literatura Latino Americano e do Caribe em Ciências da Saúde), SciELO (Scientific Eletronic Library On-line) e BDENF(Banco de Dados em Enfermagem), tendo por descritores Decs: "educação continuada", "unidade de terapia intensiva", "enfermagem", "educação permanente". Estabeleceu como critérios de inclusão os artigos publicados nacionalmente, nos período compreendido entre 2000 a 2011, com uma amostra de 21 artigos. Resultados: Desse modo, a análise dos dados possibilitou identificar muita semelhança entre os estudos; o ano que obteve maior publicação foi 2006 com (19,04\%), sendo a maioria dos pesquisadores (46.66\%) com doutorado em enfermagem. Revelou também que o maior número de artigos publicados sobre o tema proposto, foi o estado de São Paulo, com 52,38\% dos 21 artigos analisados, o que se justifica pelo fato de São Paulo ser uma região de referência na área acadêmica e de Saúde. Além disso, foi possível constatar que a unidade de terapia intensiva é um cenário para o qual toda a equipe multiprofissional deverá estar capacitada e qualificada a fim de exercer e executar suas funções com mais segurança. Considerações Finais: Acredita-se que o estudo fornecerá subsídio para a melhoria e a reflexão frente à tamanha importância da educação continuada voltada para a unidade de terapia intensiva.
\end{abstract}

Palavras-chave: Educação continuada. Unidade de terapia intensiva. Enfermagem. Educação permanente.

\begin{abstract}
Background: Continuing education is a dynamic process of teaching and learning, active and permanent intended to update and improve the training of people. Objective: This study aimed to review the existing literature about the importance of continuing education in the intensive care unit-ICU, an also to reveal the year of published articles, authors and states in which they were published. Methods: This is a qualitative quantitative, descriptive study performed by literature review published in the databases: LILACS (Latin American and Caribbean Health Sciences), SciELO (Scientific Electronic Library Online) and BDENF (the Database of Nursing), with the descriptors Decs "continuous education", "intensive care unit", "nursing", "permanent education." It established as criteria of inclusion the articles published nationwide in the period from 2000 to 2011, with a sample collection of 21 articles. Results: Therefore the analysis of the data enabled the identification of many similarities between the studies, the year which obtained the highest publication was 2006 (19.04\%), and the majority of researchers (46.66\%) with a PhD in nursing. It also revealed that the largest number of articles published about the proposed theme had been in the state of São Paulo, as compared to the other regions, with $52.38 \%$ of the 21 analyzed articles. This is justified by the fact that the state of São Paulo is a reference to the area in terms of academic life and health. . Additionally, it was found that the intensive care unit is a scenario where the whole multidisciplinary team should be trained and qualified to perform their duties and exercise with greater confidence. Conclusion: It is believed that the study will provide subsidy for the improvement and reflection front of such a great importance of continuous education focused on the intensive care unit.
\end{abstract}

Keywords: Continuous education. Intensive care unit. Nursing. Permanent education

\section{INTRODUÇÃO}

A unidade de terapia intensiva (UTI) é um cenário desafiador, para todos os profissionais, uma vez que contém equipamentos cada vez sofisticados e abrange muitos procedimentos invasivos e complexos, exigindo que o profissional se mantenha atualizado para incorporar, continuamente, novos conhecimentos e inovações tecnológicas relacionadas à UTI. Nesta unidade, estão pacientes hemodinamicamente instáveis, que necessitam de equipamentos e cuidados adequados para seu tratamento ${ }^{1,2}$.

Pinho \& Santos ${ }^{3}$, afirmam que nas instituições de alta complexidade, suas dependências devem ser providas adequadamente de estruturas físicas, recursos materiais e profissionais capacitados a realizar os cuidados efetivos em conformidade com a demanda e a especialidade.

Segundo Nascimento \& Trentini ${ }^{4}$, a UTI é um setor agitado, com inúmeras aparelhagens, em que os pacientes que neles se encontram necessitam de cuidados intensivos e dependem da tecnologia de aparelhos para sobreviverem.

A tecnologia utilizada na UTI exige que os profissionais se mantenham permanentemente atualizados acerca das habilidades técnicas aplicadas no cuidado ao paciente e também busquem conhecimento sobre o manuseio das máquinas que compõem o aparato tecnológico típico deste

Correspondência: Laurindo Pereira de Souza. Departamento de Enfermagem da Faculdade de Ciências Biomédicas (FACIMED). Av: Pedro Kemper 3660 PQ. Alvorada, Cacoal/RO. CEP: 76961-591. Telefone: 69 81121081. E-mail: laurindosorrisox@hotmail.com

Conflito de interesse: Não há conflito de interesse por parte de qualquer um dos autores.

Recebido em: 13 Jan 2015; Revisado em: 28 Jan 2015; Aceito em: 23 Fev 2015. 
setor fechado. Como novas tecnologias surgem todos os dias, faz-se necessário um trabalho constante de formação e capacitação dos profissionais que atuam neste setor, para que tomem decisões seguras e saibam utilizar, da melhor forma, os equipamentos disponíveis ${ }^{5,6}$.

A educação continuada é um processo dinâmico de ensinoaprendizagem, ativo e permanente, destinado a atualizar e melhorar a capacitação de pessoas, ou grupos, face à evolução científico-tecnológica, às necessidades sociais e aos objetivos e metas institucionais ${ }^{8}$.

Ricaldoni \& Sena ${ }^{7}$ relatam que a educação tem sido considerada como instrumento para mudanças. Afirmam ainda que, no âmbito da Educação e da Saúde, a acumulação do conhecimento, traduzido em tecnologias e indicadores de qualidade dos processos de trabalho, tem influenciado a organização do trabalho, exigindo que os trabalhadores adquiram novas habilidades de forma dinâmica.

Segundo Silva \& Seiffert ${ }^{9}$, a educação continuada é um conjunto de práticas usuais que objetivam mudanças pontuais nos modelos hegemônicos de formação e atenção à Saúde. É um processo que busca proporcionar ao indivíduo a aquisição de conhecimentos, para que ele atinja sua capacitação profissional e desenvolvimento pessoal, considerando a realidade institucional e social.

Pascoal, Montovani e Méier ${ }^{10}$ relataram em seu estudo no ano de 2007, que uma efetiva educação continuada, faz-se necessário direcioná-la ao desenvolvimento global de seus integrantes e da profissão, tendo como meta a melhoria da qualidade da assistência de enfermagem. Assim, essa tarefa não se resume a ensinar, pois engloba desenvolver no profissional de enfermagem uma consciência crítica e a percepção de que ele é capaz de aprender sempre, por meio da educação continuada e/ou permanente, e motivá-lo a buscar, na sua vida profissional, situações de ensino-aprendizagem.

Girade, Cruz, Stefanelli ${ }^{11}$ entendem que a educação continuada significa a aquisição progressiva de competências, que devem ser visíveis na qualidade do exercício da assistência de enfermagem.

Nesse sentido, torna-se fundamental então, o desenvolvimento de programas educacionais em instituições e que venham a contribuir para a melhoria da qualidade dos cuidados de enfermagem, preparando, dessa forma, profissionais capacitados a dar suas contribuições à sociedade e à unidade de terapia intensiva ${ }^{12}$.

Sendo assim, é imprescindível que o enfermeiro assuma a responsabilidade pela educação contínua de sua equipe, implementando ações que possam melhorar o padrão de assistência prestada no hospital e na comunidade, promovendo a valorização dos recursos humanos em saúde.

É importante ainda salientar que este estudo constitui extrema relevância no campo da enfermagem, pois representa uma possibilidade de contribuição ao serviço de educação continuada nas instituições, principalmente nos setores de alta complexidade como a UTI.

Diante do exposto, o objetivo desta pesquisa foi fazer uma revisão bibliográfica acerca da importância da educação continuada em unidade de terapia intensiva-UTI, revelar o ano dos artigos publicados, autores e em estados onde foram publicados.

\section{MATERIAL E MÉTODOS}

Trata-se de um estudo quantiqualitativo, descritivo realizada por meio de revisão bibliográfica, com a finalidade de analisar as produções acerca da importância da educação continuada em unidades de terapia intensiva.

A pesquisa bibliográfica tem como objetivo procurar explicar um problema a partir de referências teóricas publicadas em documentos, podendo ser, desenvolvida a partir de material já elaborado, constituído principalmente de livros, artigos científicos, dissertações de mestrados e teses de doutorado ${ }^{13}$.

O estudo descritivo identifica, sistematiza e classifica as características dos fenômenos. Por meio desta dinâmica surgem novos estudos descritivos que procuram explicar esses fenômenos, ou seja, analisar as variáveis que influenciam ou causam o surgimento dos fatos ${ }^{14}$.

Dessa forma, este tipo de estudo visa proporcionar um maior conhecimento para o pesquisador acerca do assunto, a fim de que ele possa formular problemas mais precisos ou criar hipóteses que possam ser pesquisadas por estudos posteriores $^{14}$

Os dados foram coletados em revistas e periódicos disponíveis na internet, por meio da busca em base de dados: LILACS(Literatura Latino Americano e do Caribe em Ciências da Saúde), SciELO(Scientific Eletronic Library On-line) e BDENF(Banco de Dados em Enfermagem), tendo por descritores Decs: "educação continuada", "unidade de terapia intensiva", "enfermagem", "educação permanente". Ao final, evidenciamos 20 artigos científicos e 1(uma) dissertação de mestrado, relacionados ao tema ora proposto, publicados em periódicos entre os anos de 2000 a 2011.

\section{REVISÃO DA LITERATURA}

\section{Educação}

É sabido que, para proporcionar uma assistência de enfermagem individualizada e que atenda às necessidades dos pacientes críticos, exige muita experiência ou um treinamento especializado. Pensando nisso, e em toda evolução e avanço tecnológico das unidades de terapia intensiva, julgou-se necessário aprofundar o assunto sobre a importância da educação continuada em unidade de terapia intensiva, fazendo necessário iniciar resgatando alguns conceitos de educação. 
Por meio do estudo realizado por Flores \& Ilha ${ }^{15}$, a educação faz parte de um processo pelo qual a sociedade atua intimamente sobre o desenvolvimento do indivíduo, a fim de integrá-lo ao modo de ser vigente. Assim, podemos dizer que este processo é político, situado no tempo e no espaço. Ao passo disso, a sociedade influencia e é influenciada pela situação social. O crescimento da educação nos faz ter uma visão crítica, vindo a ser decorrente de um longo processo histórico.

Freire $^{16}$, afirma que "o homem deve ser sujeito de sua própria educação, não pode ser objeto dela", portanto, a educação implica busca contínua do homem, como um ser ativo na construção do seu saber, devendo recusar as posições passivas. Nesse sentido, o homem responsabiliza-se por sua educação, procurando meios que o levem ao crescimento e ao aperfeiçoamento de sua capacidade.

A Lei de Diretrizes e Bases da Educação Nacional - LDB 9394/96, de 20 de dezembro de 1996, em seu Art. $1^{\circ}$, diz que "a educação abrange os processos formativos que se desenvolvem na vida familiar, na convivência humana, no trabalho, nas instituições de ensino e pesquisa, nos movimentos sociais e organizações da sociedade civil e nas manifestações culturais". Então, nessa perspectiva, a educação constitui parte da vida das pessoas, pois, começa desde o nascimento, num processo contínuo, dinâmico e interativo, buscando cada vez mais características, que fundamentam a educação ${ }^{18}$.

O Código de Ética dos Profissionais de Enfermagem (CEPE) ${ }^{22}$ no capítulo dos direitos e das responsabilidades dos profissionais no seu art.14 diz que: "O profissional tem o direito de atualizar seus conhecimentos técnicos, científicos e culturais, em benefício da clientela, da coletividade e do desenvolvimento da profissão".

Já o Decreto17 94.406, de 25 de junho de 1986, que regulamenta o exercício da enfermagem, especifica claramente que ao enfermeiro incumbe, como integrante da equipe de saúde, a "participação nos programas de treinamento e aprimoramento de pessoal de saúde, particularmente nos programas de educação continuada" (Art. $11^{\circ}$ ). Sendo assim, além do direito e dever ético de manter-se atualizado, o enfermeiro, independentemente da função que desempenha, tem a obrigação legal de ser facilitador do processo educativo, para os demais membros da equipe de enfermagem.

\section{Educação continuada e permanente}

Para Silva, Conceição \& Leite $^{8}$, a educação continuada é um componente essencial dos programas de formação e desenvolvimento de recursos humanos das instituições.

De acordo com Oguisso ${ }^{23}$, a Organização Panamericana de Saúde (OPAS) revela que a educação continuada é um processo dinâmico de ensino-aprendizagem, ativo e permanente, destinado a atualizar e melhorar a capacitação de pessoas ou grupos, face à evolução cientifico-tecnológica, às necessidades sociais e aos objetivos e metas institucionais.

J. Health Biol Sci. 2015; 3(1):39-45
Corroborando com essa afirmação a Organização Mundial de Saúde (OMS) reconhece a educação continuada como essência para a qualidade da assistência à saúde ${ }^{20}$.

Diante de tamanha importância, as pesquisas ${ }^{19,10}$ revelaram que as ações de educação continuada proporcionam, acima de tudo, o desenvolvimento de um processo educativo que possibilita a reflexão do "por que fazer", "para que fazer" e "para quem fazer", levando os profissionais a buscar coletivamente alternativas para mudanças substanciais na prática cotidiana visando atender às necessidades de saúde da população, interagindo e intervindo e, nesse sentido, a formação deve ter como características: a autonomia e a capacidade de aprender constantemente, de relacionar teoria e prática e vice-versa, isto se refere à inseparabilidade do conhecimento e da ação.

Corroborando ainda com essa temática, esses autores ${ }^{9,24}$, relatam em seus estudos que a importância da educação continuada para a enfermagem, é prioritária e dentro dessa educação contínua, estão os programas de inclusão, atualização, treinamento, pós-graduação, pesquisa, eventos, produção, gerência e integração docência-assistência - todos conduzidos e fundamentados no cuidado humano e no cuidado profissional da enfermagem.

Esta outra investigação ${ }^{25}$ revelou que a educação permanente é um processo educativo que ocorre no espaço do pensar e do fazer do trabalho. Tem como desafio estimular o desenvolvimento dos profissionais sobre um contexto de responsabilidades e necessidades de atualização, uma vez que, é um processo de reflexão, de mudanças e transformações, considerando, para isso o serviço, o trabalho, o cuidado, a educação e a qualidade da assistência.

Entretanto, esses estudiosos ${ }^{25,27}$, entendem que a educação permanente, continuada, pode motivar a transformação pessoal e profissional do sujeito, buscando alternativas para minimizar as dificuldades existentes na realidade de ensino no hospital, inclusive nas unidades de cuidados de pacientes críticos.

\section{A unidade de terapia intensiva (UTI)}

As unidades de terapia intensiva surgiram como resposta ao problema do tratamento aos pacientes graves, tornando-se áreas hospitalares destinadas aqueles em estado crítico que necessitam de cuidados altamente complexos. O serviço de enfermagem nas UTIs, foi marcado por esforços inicias para promover eficiência por meio de escolhas e seleção de algumas práticas seguras na assistência ao paciente grave, sujeitos à instabilidade hemodinâmica e cujo tratamento requer apoio de equipamento especial, de diagnóstico e tratamento. Diante disso, surge o desafio de adaptar os novos funcionários ao local de trabalho, que é cada vez mais complexo ${ }^{1,2,6}$.

Para entender a dinâmica na qual as ocorrências iatrogênicas acontecem, implica em visualizar a segurança do paciente mantido como um todo, um sistema abrangente e complexo, composto por vários subsistemas que se interligam e 
interagem entre si, contribuindo em seu conjunto para uma maior segurança do paciente. Revela então, a importância do enfermeiro na UTI, ter o papel de planejar, orientar e aplicar uma assistência contínua com qualidade que gere segurança ao paciente e à sua equipe ${ }^{2}$.

Conforme esses pesquisadores ${ }^{31,33}$ a UTI é equipada de tanta tecnologia avançada, e isso faz que o profissional se distancie do paciente, dando mais atenção às sinalizações das máquinas e esquecendo o que é mais importante: os valores humanos. No entanto, nota-se nos estudos de Schwonke ${ }^{30}$ que essa relação de cuidados entre pacientes e máquinas é bem entendida pelos profissionais, pois há maiores chances de efeitos terapêuticos satisfatórios e melhora no prognóstico do paciente.

Silva \& Ferreira ${ }^{32}$ mostraram em seu estudo publicado no ano de 2011 pela Revista Brasileira de Enfermagem (REBEn) que o grande arsenal tecnológico nas UTIs utilizados pelos enfermeiros para auxiliar na manutenção da vida é uma realidade que, ao mesmo tempo em que encanta, também assusta sendo um grande desafio, pois programar máquinas, ajustar parâmetros, supervisionar alarmes e seu funcionamento requer do profissional um conhecimento teórico-prático científico específico cada vez maior e nesse encontro é que se exige que todas as unidade de terapia intensiva devem ter programas funcionantes de educação continuada permanente.

\section{ANÁLISE E DISCUSSÃO DOS DADOS}

Após a leitura dos artigos, foi possível identificar a visão de diversos autores a respeito da temática.

Segundo Nishiyama, Beccaria \& Ittavo ${ }^{28}$ a importância da educação continuada é evidente, pois muitas unidades de terapia intensiva não assumem essas responsabilidades, devido à existência de dificuldade para implantação de programas adequados. Afirmam ainda que o profissional da enfermagem deve ter consciência que é um direito dele e dever da instituição de saúde trabalhar a favor do seu desenvolvimento.

Pesquisas ${ }^{24,32}$ revelam que é por meio da educação continuada na unidade de terapia intensiva que o profissional enriquece seus conhecimentos teóricos e científicos; sendo assim, ele afirma o quanto cuida com qualidade.

Nesse contexto, a educação continuada leva ao entendimento de que o indivíduo deve ter uma meta a ser seguida por toda sua vida, buscando garantir sempre a sobrevivência profissional e da enfermagem, por meio de competências, conhecimento e capacitações relacionados aos cuidados a pacientes críticos $^{2,4-6,15,21}$.

A equipe de enfermagem constitui a equipe mais ampla do setor da saúde, e a cada dia esta aumentando, embora cada formação com sua heterogeneidade diversificada. Portanto, a educação profissional deve ser um processo que propicie novos conhecimentos, que capacite para execução adequada do trabalho e que prepare para futuras oportunidades de ascensão profissional $^{26}$.

É nesse sentido que a unidade de terapia intensiva por ser um setor completo e complexo exige que cada profissional que ali atue, passe por um processo de capacitação e atualização de seus conhecimentos científicos e práticos, para que sua assistência seja prestada com qualidade e segurança, ao paciente envolvido em tanta tecnologia ${ }^{1-4,6}$.

Segundo alguns autores ${ }^{6,25,27}$, a educação continuada em unidade de terapia intensiva e prioritária, pois é por meio dela que a capacitação e treinamento ocorrem visando, assim, reduzir os eventos adversos, promovendo também maior segurança ao paciente que, na maioria das vezes é totalmente dependente da enfermagem.

Entretanto Paschoal ${ }^{29}$, ao relacionar essa concepção de educação com a profissão de enfermagem, considerada também prática social, compreende que, em todas as ações de enfermagem, estão inseridas ações educativas. Dessa forma, há necessidade de se promoverem efetivas oportunidades de ensino, fundamentadas na conscientização do valor da educação como meio de crescimento dos profissionais da enfermagem, assim como o reconhecimento deles pela função educativa no desenvolvimento do processo de trabalho.

Ciampone \& Perez ${ }^{21}$, corroboram que a educação continuada em saúde é utilizada como política de formação e qualificação de profissionais em que o processo educativo deve ser dinâmico, contínuo e trazer mudanças e transformações sociais e práticas. Assim, deve acompanhar os avanços tecnológicos e socioeconômicos, bem como, articular o aprendizado e a reflexão crítica. Este autor ${ }^{25}$, afirma a importância de ser contínua e permanente a capacitação em unidade de terapia intensiva, pois a carência de aprender e transformar são inerentes à condição de ser humano. Compreende-se que, em todo o seu processo de trabalho, estão inseridas ações educativas.

Para Viana ${ }^{6}$, o trabalho em UTI é intenso e exaustivo, devendo o enfermeiro estar preparado e capacitado para identificar e atender qualquer instabilidade que os clientes ali internados possam apresentar, intensificando, assim, a busca por informação. Souza, Mota \& Mota ${ }^{33}$, afirmam em seus estudos que a UTI tem sido vista como um cenário frio, hostil e estressante, em que a equipe realiza procedimentos de forma mecânica, apagando de seu psíquico que ali existem seres humanos que precisam de cuidado e atenção.

Tão relevante e ao mesmo tempo importante é o assunto sobre educação continuada e permanente na unidade de terapia intensiva que, em 2003, o Ministério da Saúde torna a Educação Permanente uma política de saúde do Sistema Único de Saúde (SUS), com a criação dos Polos de Educação Permanente em Saúde ${ }^{19}$.

Para uma melhor compreensão didática, a tabela 1(um) mostra o ano de publicação dos autores dos artigos revisados. 
Tabela 1. Ano dos artigos publicados referente a educação continuada na unidade de terapia intensiva no período de 2000 a 2011.

\begin{tabular}{lrr}
\hline Item & $\mathbf{N}^{\circ}$ de artigos & $\%$ \\
\hline 2000 & 2 & 9,52 \\
2001 & 1 & 4,76 \\
2002 & 1 & 4,76 \\
2003 & 0 & 0,00 \\
2004 & 2 & 9,52 \\
2005 & 0 & 0,00 \\
2006 & 4 & 19,04 \\
2007 & 1 & 4,76 \\
2008 & 2 & 9,52 \\
2009 & 3 & 14,30 \\
2010 & 2 & 9,52 \\
2011 & 3 & 14,30 \\
\hline Total & $\mathbf{2 1}$ & 100,00 \\
\hline
\end{tabular}

Dos artigos encontrados no período de 2000 a 2011, percebe-se que houve um número maior de publicações no ano de 2006, com $19.04 \%$ referentes ao tema proposto. Ainda percebemos outros aumentos nos anos de 2009 e 2011 com 14,30\% que se justificam pelo fato de as inovações tecnológicas dentro das unidades de terapia intensiva terem aumentado, com o acréscimo de aparelhagens mais modernas que surgem a cada dia e que exigem mais capacitação e treinamento da equipe que atua na UTI.

A tabela 2(dois) mostra a formação dos profissionais que participaram das publicações dos artigos revisados.

Tabela 2. Formação dos autores que produziram artigos referentes à educação continuada em unidade de terapia intensiva no período de 2000 a 2011.

\begin{tabular}{llr}
\hline \multicolumn{1}{c}{ Profissionais } & № & \% \\
\hline Enfermeira(o) & 05 & 16,68 \\
Mestre em enfermagem & 10 & 33,33 \\
Doutor em enfermagem & 14 & 46,66 \\
Doutorando em enfermagem & 01 & 3,33 \\
\hline \multicolumn{1}{c}{ Total } & $\mathbf{3 0}$ & $\mathbf{1 0 0 , 0 0}$ \\
\hline
\end{tabular}

Percebe-se que houve maior interesse por parte dos enfermeiros doutores em pesquisar o assunto, um total de $46.66 \%$ dos 30 profissionais envolvidos na pesquisa dos artigos revisados. Este maior número de publicações envolvendo o tema abordado por esses especialistas, deve-se ao fato de eles estarem coordenando grupos de pesquisas científicas, referentes à unidade de terapia intensiva, e programas de educação continuada e permanente.

Conclui-se que, quanto maior o nível de titulação, mais chance terão os profissionais de se envolverem em pesquisas científicas, como a coordenação de projetos de extensão e pesquisa, entre outras.

A tabela 3 (três) mostra os locais das publicações dos artigos encontrados referentes à importância da educação continuada em unidades de terapia intensiva.

Tabela 3. Locais onde os artigos foram publicados no período de 2000 a 2011.

\begin{tabular}{lrr}
\hline \multicolumn{1}{c}{ Item } & $\mathbf{N}^{\circ}$ de Artigos & $\mathbf{\%}$ \\
\hline São Paulo & 11 & 52,38 \\
Santa Catarina & 01 & 4,76 \\
Rio de Janeiro & 02 & 9,52 \\
Paraná & 01 & 4,76 \\
Porto Alegre & 02 & 9,52 \\
Distrito Federal & 03 & 14,30 \\
Goiás & 01 & 4,76 \\
\hline Total & $\mathbf{2 1}$ & $\mathbf{1 0 0 , 0 0}$ \\
\hline
\end{tabular}

Houve um maior número de artigos publicados sobre o tema proposto, no estado de São Paulo do que em outras regiões, somando um total de $52.38 \%$ dos 21 artigos analisados, conforme mostra a tabela acima. Isso se justifica pelo fato de São Paulo ser uma região de referência para a área acadêmica e da Saúde, seguida por Brasília com $14.30 \%$ dos artigos revisados referente à educação continuada na unidade de terapia intensiva.

A diminuição no número de pesquisas ou mesmo a omissão de alguns estados não publicarem artigos referentes ao tema no período abordado, é consequência da falta de investimento em políticas de incentivo a pesquisas nessas regiões.

\section{CONSIDERAÇÕES FINAIS}

Existe a necessidade de buscar a participação de todos os envolvidos nas questões educativas na enfermagem: educadores, instituições, políticos, meios econômicos e sociais, para que sua relação de troca, indispensável à prática profissional, alcance o desenvolvimento pessoal e profissional. Assim, considera-se que a educação continuada constitui uma oportunidade para capacitação e a atualização em unidade de terapia intensiva, além de propiciar melhoria na qualidade da assistência e segurança ao paciente grave, visto que mobiliza também outros membros da equipe multidisciplinar e não somente a enfermagem.

Conclui-se, nessa pesquisa, que a unidade de terapia intensiva exige profissionais capacitados e preparados técnica e cientificamente, com conhecimento aprofundado, para que possa manusear todo o arsenal tecnológico existente dentro da UTI, sem trazer risco para o cliente, garantindo-Ihe total segurança.

Foram analisados neste estudo 21 artigos científicos em sua 
totalidade. Dispomos de 3 (três) tabelas, as quais mostram o ano de publicação dos artigos, profissionais e locais e onde foram publicados os respectivos artigos sobre a importância da educação continuada em unidades de terapia intensiva, todos apresentados em porcentagem para uma melhor compreensão. Conclui-se, também, que o profissional que mais publicou artigos referentes ao tema proposto, tinha como titulação um doutorado em enfermagem e coordenava programas de educação continuada ou outros programas voltados para a educação e para pesquisas científicas em saúde.

Torna-se evidente a necessidade de implantação e criação de programas de educação continuada e de educação permanente, assim como a literatura revela que são extremamente importantes para o profissional que atua na UTI, bem como a educação em serviço, que já vem incorporada a esses programas.

Evidencia-se em nossa pesquisa a existência de poucas publicações referentes ao tema e também pouco interesse dos profissionais da área em pesquisar o assunto em questão, visto ser um assunto de extrema complexidade; igualmente, que as unidades de terapias intensivas vêm aumentando gradativamente nos dias atuais. Acreditamos que o estudo fornecerá subsídio para a melhoria e a reflexão em face da grande importância da educação continuada voltada para as unidades de terapia intensiva.

\section{REFERÊNCIAS}

1. Knobel E. Condutas no paciente grave. São Paulo: Atheneu; 2006.v.1.

2. Padilha KG. org. Enfermagem em UTI: cuidando do paciente critico. São Paulo: Manole; 2010.

3. Pinho LB; Santos SMA. Dialética do cuidado humanizado na UTI: Contradições entre o discurso e pratica profissional do enfermeiro. Revesc. enferm. USP. 2008 [acesso 2013 ago 10]; 42(1):66-72. Disponível em: http://www.scielo.br/scielo. php?script=sci_arttext\&pid=S0080-62342008000100009\&lng=en. doi: http:// dx.doi.org/10.1590/s0080-62342008000100009.

4. Nascimento ERP; Trentini M. O cuidado de enfermagem na unidade de terapia intensiva (UTI): teoria humanística de Paterson e Zderad. Rev LatinoAm Enfermagem. 2004 mar-abr; 12(2):250-7. doi:.http://dx.doi.org/10.1590/ S0104-11692004000200015.

5. Godinho JSL. A Educação permanente na UTI neonatal: pesquisa exploratória de campo [dissertação]. Rio de Janeiro: Universidade Federal Fluminense; 2009.

6. Viana RAPP, organizador. Enfermagem em terapia intensiva: práticas e vivências. Porto Alegre: Artmed; 2011.

7. Ricaldoni CAC, Sena RR. Educaçao permanente: uma ferramenta para pensar e agir no trabalho de enfermagem. Rev Latino-Am. Enfermagem [internet]. 2006 nov-dez [acesso 2013 jun 20]; 14(6):837-842. Disponível em:http://www.scielo. $\mathrm{br} /$ scielo.php?script=sci_arttext\&pid=S0104-11692006000600002\&lng=en doi: http://dx.doi.org/10.1590/S0104-11692006000600002.

8. Silva MF, Conceição FA, Leite MMJ. Educação continuada: um levantamento de necessidade da equipe de enfermagem. Arq. BrasCiên Saúde. 2009 jan- abr; 34(1):15-20.

9. SilvaGM, Seiffert, OMLB. Educação continuada em enfermagem: Uma proposta metodológica. Rev bras enferm. 2009, maio-jun; 62(3):362-6.doi: http://dx.doi.org/10.1590/S0034-71672009000300005.

10. Paschoal AS; Montovani MF; Méier MJ. Percepção da educação permanente, continuada e em serviço para enfermeiros de um hospital de ensino. RevEscEnferm USP. 2007 set; 41(3):478-84.doi: .http://dx.doi.org/10.1590/ S0080-62342007000300019.

11. Girade MG; Cruz EMNT; Stefanelli MC. Educação continuada em enfermagem psiquiátrica: reflexão sobre conceitos. Rev. esc. enferm. USP. 2006 mar; 40(1). doi:http://dx.doi.org/10.1590/S0080-62342006000100015.

12. Darvim RMB; Torres GV; Santos SR. Educaçao continuada em enfermagem: conhecimentos atividades e barreiras encontradas em uma maternidade escola. Rev Latino-am Enfermagem. 1999 dez. doi: http://dx.doi.org/10.1590/ S0104-11691999000500006.
13. Cervo AL,Berviam PA. Metodologia cientifica. 4.ed. São Paulo: Macron Books;1996.

14. Gil AC. Como elaborar projetos de pesquisa. 3.ed. São Paulo: Atlas; 1999.

15. Flores LMM; Ilha NLP. Educação continuada em enfermagem. DisciplinarumScientia. 2001;2(1): p. 79-86.

16. Freire P. Educação e mudança. Rio de Janeiro: Paz e Terra; 2001.

17. Brasil. Lei 7498 , de 26 de junho, 1986. Dispõe sobre a regulamentação do exercício da enfermagem e dá outras providências. Diário Oficial da União, Diário Ofical [da] República Federativa do Brasil.1986 jun. 26; Seção 1. p. 9273.

18. Brasil. Ministério da Educação. Lei no 9397 de 20 de dezembro de 1996. Estabelece as diretrizes e bases da educação nacional. Diário Ofical [da] República Federativa do Brasil. 1990 dez 20; Seção 1. p 27833.

19. Ministério da Saúde (Brasil), Secretaria de Gestão do trabalho e da Educação na Saúde, Departamento de Gestão de educação Permanente na Saúde. Política de educação e desenvolvimento para o SUS: caminhos para a educação permanente em saúde: pólos de educação permanente em saúde. Brasília: Ministério da Saúde; 2004

20. Ministério da Saúde (Brasil), Secretaria de Modernização Administrativa e Recursos Humanos. A educação continuada de enfermeiros do SUS. Brasília: Centro de Documentação; 1990.

21. Ciampone MHT; Peres AM. Gerência e competências gerais do enfermeiro. Texto Contexto Enferm. 2006 jul-set; 15(3): 492-499. doi: .http://dx.doi. org/10.1590/S0104-07072006000300015.

22. Conselho Regional de Enfermagem de Rondônia. Caderno de Legislação. 7. ed. Porto Velho: COREN; 2011.

23. Oguisso TA. Educação continuada como fator de mudanças: visão mundial. Rev. Nursing. 2000; 3(20):22-9.

24. Paschoal AS,Montovani MF, Lacerda MR. A educação permanente em enfermagem: subsídios para a prática profissional. RevGauchaEnferm. 2006 set; 27(3):336-43.

25. Jesus MCP, Figueiredo MAG, Santos SMR, Amaral, AMM, Rocha, LO, Thiollent MJM. Educação permanente em enfermagem em um hospital universitário. Rev escenferm USP. 2011 oct;45(5):1229-36. doi:http://dx.doi.org/10.1590/S008062342011000500028

26. Kurcgant $P$, organizador. Administração em enfermagem. São Paulo: EPU; 1991. 
27. Montanha D,Peduzzi M. Educação permanente em enfermagem: levantamento de necessidade e resultado esperados segundo a concepção dos trabalhadores. Ver escenferm USP. 2010 set;44(3):597-604. doi:http://dx.doi. org/10.1590/S0080-62342010000300007.

28. Nishiyama K, Beccaria LM,Ittavo J. Enfermeiros recém admitidos em UTI: a importância de programas de educação que possibilitem a sua inserção.. In: Anais do 8. Simpósio Brasileiro de Enfermagem Comunicação [internet]; 2002 May 02-03; São Paulo, SP, Brasil; 2002 [acesso 2014 maio 25]. Disponível em: http://www.proceedings.scielo.br/scielo.php?script=sci_ arttext\&pid=MSC0000000052002000100030\&Ing=en\&nrm=van

29. Paschoal AS. O discurso do enfermeiro sobre educação permanente no grupo focal. [dissertação]. Curitiba (PR): Universidade Federal do Paraná; 2004.

30. Schwonke CRGB, Lunardi WD, Filho, Lunardi VL,Santos SSCS, Barlem ELD. Perspectivas filosóficas do uso da tecnologia no cuidado de enfermagem em terapia intensiva. Ver bras enferm. 2011 jan-fev; 64(1):189-192. doi: http:// dx.doi.org/10.1590/S0034-71672011000100028.

31. Silva RCL, Porto IS, Figueiredo NMA. Reflexões acerca da assistência de Enfermagem e o discurso de humanização. Esc Anna Nery Rev Enferm. 2008 mar; 12(1):156-9.

32. Silva RC, Ferreira MA. Características dos enfermeiros de uma unidade tecnológica: implicações para o cuidado de enfermagem. Ver Bras Enferm [internet]. 2011 jan-fev [acesso 2014 maio 25]; 64(1):98105. Disponível em: http://www.scielo.br/scielo.php?script=sci arttext\&pid=S003471672011000100015\&Ing=en. http://dx.doi.org/10.1590/ S0034-71672011000100015.

33. Souza SAJ. Mota CGS, Mota LKC, Espíndula MB. Assistência de enfermagem na UTI: uma abordagem holística. Revista Eletrônica de Enfermagem do Centro de Estudo de Enfermagem e Nutrição. 2010 jan-jun; 1(1): 1-16. 